\title{
Characteristic Crossing Points in Specific Heat Curves of Correlated Systems
}

\author{
D. Vollhardt* \\ Institut für Theoretische Physik C, Technische Hochschule Aachen, D-52056 Aachen, Germany
}

(Received 30 August 1996)

\begin{abstract}
Attention is drawn to the observation that in many correlated systems (e.g., ${ }^{3} \mathrm{He}$, heavy fermion systems, and Hubbard models) the specific heat curves, when plotted for different values of some thermodynamic variable (e.g., pressure, magnetic field, and interaction), cross almost precisely at one or two temperatures. A quantitative explanation of this phenomenon, based on the form and the temperature dependence of the associated generalized susceptibilities, is presented. [S0031-9007(97)02432-0]
\end{abstract}

PACS numbers: 71.27.+a, 67.55.Cx, 71.28.+d

In 1959 Brewer et al. [1] noticed that the specific heat curves $C(T, P)$ of liquid ${ }^{3} \mathrm{He}$ measured at different pressures $P$ all intersect at a temperature $T_{+} \simeq 0.16 \mathrm{~K}$, and that $C(T, P)$ increases with increasing pressure below $T_{+}$. Greywall's high-precision measurements of the specific heat at constant volume $V$ provided striking evidence for the sharpness of the crossing point at $T_{+} \simeq 160 \mathrm{mK}$ [see Fig. 1(a)] [2]. At this temperature the specific heat is obviously independent of volume or pressure. It was unclear, however, whether special significance should be attached to this finding [2,3]. Recently, Georges and Krauth [4] observed the same conspicuous crossing phenomenon in quite a different system, namely, in the paramagnetic phase of the Hubbard model, the simplest model of correlated electrons, in infinite dimensions. For small to intermediate values of the local interaction $U$ the specific heat curves $C(T, U)$, calculated by iterated perturbation theory, were found to intersect almost at the same temperature $T_{+} \simeq 0.59 t^{*}$, where $t^{*}$ is the scaled hopping amplitude of the electrons (Fig. 2) [5]. Clearly the existence of these peculiar points of intersection calls for an explanation.

In this Letter we illustrate that crossing points such as the ones described above can actually be observed in the

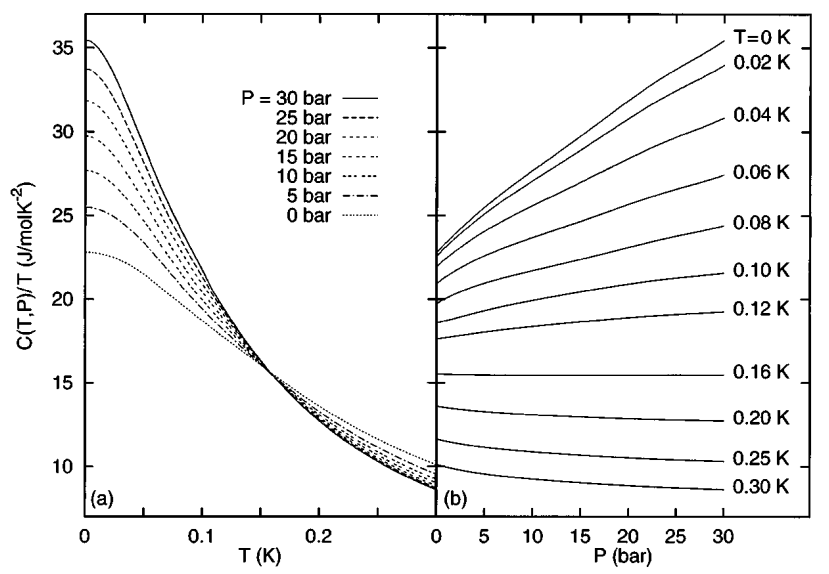

FIG. 1. Specific heat $C(T, P)$ of ${ }^{3} \mathrm{He}$ [2]: (a) $C / T$ vs $T$, (b) $C / T$ vs $P$. specific heat of many correlated systems. Furthermore, we discuss the origin of this phenomenon.

To be able to discuss the problem in a sufficiently general framework we define a general free energy $\Phi(T, X)$, where $X$ can be any thermodynamic variable (here we choose intensive variables), e.g., pressure $(P)$, magnetic field $(B)$, and on-site interaction $(U)$. The conjugate extensive variable associated with $X$ is $\xi(T, X)=$ $-\partial \Phi(T, X) / \partial X$. Pairs of variables $(X, \xi)$ are, for example, $(P,-V),(B, M)$, and $(U,-D)$, with $M$ as the magnetic moment, and $D$ as the number of doubly occupied sites in Hubbard models. With the entropy $S(T, X)=-\partial \Phi / \partial T$ one obtains the Maxwell relation

$$
\frac{\partial S(T, X)}{\partial X}=\frac{\partial \xi(T, X)}{\partial T} .
$$

A search of the literature reveals that there exists quite a number of systems, both in theory and experiment, where the specific heat curves $C(T, X)=T \partial S(T, X) / \partial T$ versus $T$ when plotted for different, not too large values of $X$ intersect at one or even two well-defined, nonzero temperatures. Apart from normal-liquid ${ }^{3} \mathrm{He}$ it can be observed in heavy fermion systems with and without Fermi liquid

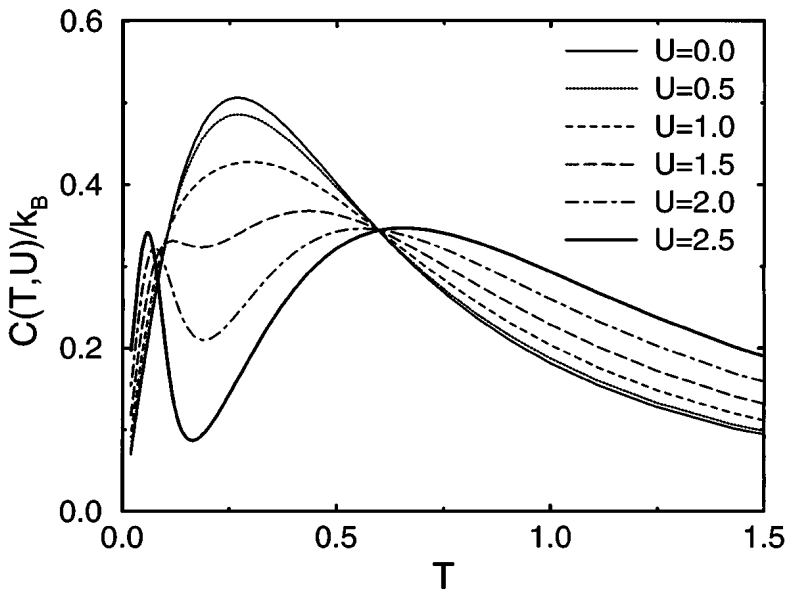

FIG. 2. Specific heat $C(T, U)$ of the paramagnetic phase of the Hubbard model in $d=\infty$ dimensions calculated by iterated perturbation theory [5]. 
behavior, for example, in $\mathrm{CeAl}_{3}$ [6] [Fig. 3(a)] and $\mathrm{UBe}_{13}$ [7] upon change of $P$, in $\mathrm{UPt}_{3-x} \mathrm{Pd}_{x}$ [8] and $\mathrm{CePtSi}_{1-x} \mathrm{Ge}_{x}$ [9] as $x$ is varied, and in $\mathrm{CeCu}_{6-x} \mathrm{Au}_{x}(0.2 \leq x \leq 0.5)$ when either $P$ [10] or $B$ [11] [Fig. 3(b)] is varied. It is also found in $\mathrm{Eu}_{0.5} \mathrm{Sr}_{0.5} \mathrm{As}_{3}$, a semimetal with competing interactions, upon change of $B$ [12]. In particular, all theoretical models of Fermi and Luttinger liquids investigated beyond the low-temperature regime show this feature: the one-dimensional $(d=1)$ Hubbard model in a magnetic field [13], the $1 / r$ Hubbard in $d=1$ in the metallic phase when the interaction $U$ is changed [14], and the Hubbard model in $d=\infty$ discussed above [4].

To explain the origin of the crossing points we separate the problem into two questions: (i) Why do specific heat curves cross at all? (ii) How wide is the region where the curves cross? Turning to the first question, we note that any crossing of specific heat curves $C(T, X)$ implies

$$
\left.\frac{\partial C(T, X)}{\partial X}\right|_{T_{+}(X)}=\left.T_{+}(X) \frac{\partial^{2} \xi(T, X)}{\partial T^{2}}\right|_{T_{+}(X)}=0 .
$$

Thus crossing occurs where $\xi(T, X)$ versus $T$ has a turning point. In general the crossing temperature $T_{+}(X)$ still depends on $X$. Only if $T_{+}$is independent of $X$ for some range of $X$ values do the curves intersect at one point. Crossing of specific heat curves may be inferred from a sum rule for the change of the entropy $S(T, X)$ with respect to $X$ in the limit $T \rightarrow \infty$

$$
\eta_{X}=k_{B}^{-1} \lim _{T \rightarrow \infty} \frac{\partial S(T, X)}{\partial \ln X}=\frac{X}{k_{B}} \int_{0}^{\infty} \frac{d T^{\prime}}{T^{\prime}} \frac{\partial C\left(T^{\prime}, X\right)}{\partial X} .
$$

1. Lattice models $(X \equiv U)$. - Equation (3) implies $\eta_{U}=0$ for any kind of Hubbard model since $S$ ap- proaches a constant for $T \rightarrow \infty$. At high temperatures, $T \gg U, C(T, U) \propto U / T$, i.e., $\partial C / \partial U>0$. Hence $\partial C / \partial U$ must become negative at intermediate temperatures, i.e., the specific heat curves must cross at least at one temperature, for the integral to vanish identically. We note that this is a genuine correlation effect originating from the existence of $U^{2}$ and higher terms in an expansion of the internal energy $E(T, U)$, and hence of $C(T, U)=\partial E / \partial T$, in powers of $U$.

2. Continuum systems $(X \equiv P)$. - Here Eq. (3) implies $\eta_{P}=-1$ since $S$ approaches the ideal-gas value for $T \rightarrow \infty$. Apparently $\partial C / \partial P<0$ at most (especially high) temperatures [15].

In the Fermi liquid phase of ${ }^{3} \mathrm{He}[1,2]$ and in the paramagnetic phase of the Hubbard model at low temperatures $[4,13]$ the entropy is known to increase with $X(\equiv P, U)$, i.e., $\partial C / \partial X>0$. At sufficiently low $T$, when $S(T, X)=$ $C(T, X)=\gamma T$, this implies $d \gamma / d X>0$. This may be attributed to the excitation of low-energy (spin) degrees of freedom in the correlated system [16]. Hence, the specific heat curves will cross at some low temperature $T_{+}(X)$. (In ${ }^{3} \mathrm{He} T_{+}$practically coincides with the temperature above which Fermi liquid theory breaks down [17].) Equation (3) then implies that in the Hubbard model the specific heat curves will cross twice altogether (Fig. 2). These two systems only consist of a single species of particles. By contrast, heavy fermion systems are basically two-component systems consisting of conduction and localized electrons which may hybridize. The strength of the hybridization is determined by an amplitude $V_{\text {hyb }}(P)$ which increases with pressure. By hybridizing, the electrons may gain an energy $k_{B} T_{K}$, where
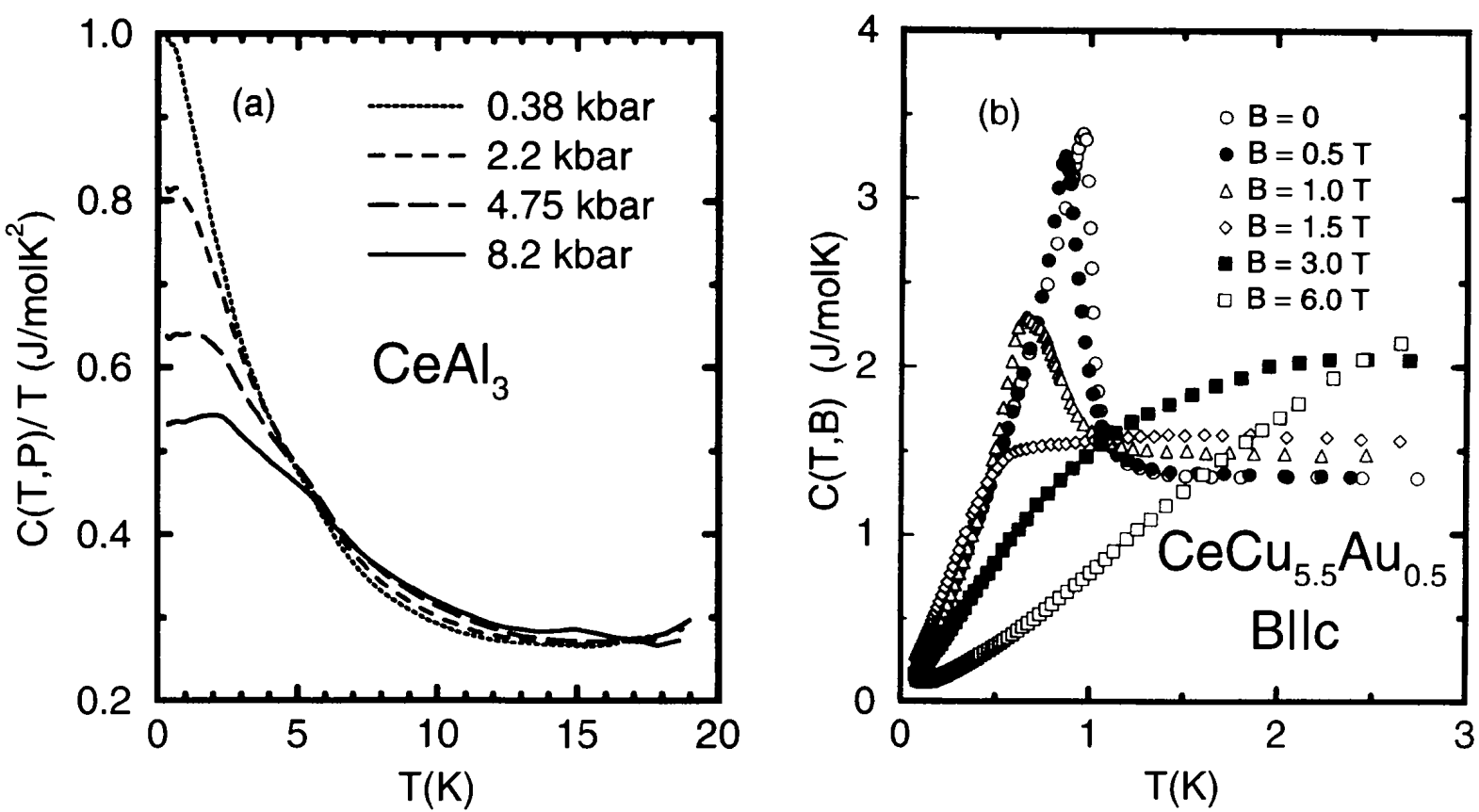

FIG. 3. Specific heat (a) $C(T, P) / T$ of $\mathrm{CeAl}_{3}$ [6] (for $T>8 \mathrm{~K}$ we took the running average of the data points to reduce the scatter), (b) $C(T, B)$ of $\mathrm{CeCu}_{5.5} \mathrm{Au}_{0.5}[11]$. 
$T_{K}(P) \propto \exp \left(-\right.$ const $\left./ V_{\text {hyb }}^{2}\right)$ is a different ("Kondo") lowenergy scale. Below $T_{K}$ the specific heat is linear, the coefficient being given by $\gamma(P) \propto 1 / T_{K}$, where now $d \gamma / d P<0$. Hence at low temperatures the specific heat decreases with pressure [18]. This is also the case at higher temperatures when crystal field excitations [19] or phonons become important. In the interval between these temperatures the specific heat must therefore increase with pressure for the entropy to be conserved. This implies that the specific heat curves will cross at two temperatures $T_{+}$and $T_{+}^{\prime}$. This is precisely what is seen in several heavy fermion systems, e.g., in $\mathrm{CeAl}_{3}[6]\left[T_{+} \simeq\right.$ $5 \mathrm{~K}, T_{+}^{\prime} \simeq 17 \mathrm{~K}$; see Fig. 3(a)] and $\mathrm{UBe}_{13}$ [7] $\left(T_{+} \simeq\right.$ $\left.2.5 \mathrm{~K}, T_{+}^{\prime} \simeq 9 \mathrm{~K}\right)$. Yet another reason for specific heat curves to cross is the vicinity of a second-order phase transition where the discontinuity in $C(T, X)$ is changed with $X$ as in $\mathrm{CeCu}_{6-x} \mathrm{Au}_{x}$ [11] with $X$ as the magnetic field.

We now turn to the question concerning the width of the crossing region. We expand $C(T, X)$ in an (asymptotic) series in $X-X_{0}$, with $X_{0}$ chosen at convenience,

$$
\begin{aligned}
C(T, X) \simeq & C\left(T, X_{0}\right)+\left.\left(X-X_{0}\right) T \frac{\partial^{2} \xi}{\partial T^{2}}\right|_{X_{0}} \\
& +\left.\frac{1}{2}\left(X-X_{0}\right)^{2} T \frac{\partial^{2}}{\partial T^{2}} \frac{\partial \xi}{\partial X}\right|_{X_{0}}+\ldots,
\end{aligned}
$$

where we used Eq. (2). At $T_{+}\left(X_{0}\right)$ Eq. (4) implies $C\left(T_{+}, X\right) \simeq C\left(T_{+}, X_{0}\right)\left[1+W_{X_{0}}(X)\right]$ where the (relative) width of the crossing region, $\left|W_{X_{0}}(X)\right|=\mid \Delta_{X_{0}}^{(1)}(X)+$ $\Delta_{X_{0}}^{(2)}(X)+\ldots \mid$, is determined by the numbers

$$
\Delta_{X_{0}}^{(n)}(X)=\left.\frac{\left(X-X_{0}\right)^{n+1} T_{+}}{(n+1) ! C\left(T_{+}, X_{0}\right)} \frac{\partial^{2}}{\partial T^{2}} \chi^{(n)}\left(T, X_{0}\right)\right|_{T_{+}},
$$

with $\chi^{(n)}(T, X)=\partial^{n} \xi / \partial X^{n}$. For $\left|\Delta_{X_{0}}^{(n)}(X)\right| \ll 1$ the $C(T, X)$ curves will intersect at a well-defined point. The width is seen to be determined by the curvature (with respect to $T)$ of the linear $(n=1)$ and nonlinear $(n>1)$ susceptibilities $\chi^{(n)}(T, X)$ at $T_{+}$and $X_{0}$. There are two particularly relevant sufficient conditions under which the $\Delta^{(n)}$ are small.

(i) Weak $T$ dependence of $\chi^{(n)}$ : For $X=P, \xi=-V$ the susceptibility $\chi^{(1)}=-\partial V / \partial P=\kappa_{T} V$ is essentially the isothermal compressibility of the system. In the strongly correlated, high-density quantum liquid ${ }^{3} \mathrm{He}$ the volume $V(T, P)$ and the change of volume with pressure $\chi^{(1)}(T, P)$ depend only very weakly on temperature for all $T \lesssim 2.5 \mathrm{~K}$ [20]. The Maxwell relation (1) then implies that the curvature of $C(T, P) / T$ vs $P$, $\partial^{2}[C(T, P) / T] / \partial P^{2}=\partial^{2} \chi^{(1)} / \partial T^{2}$, is also small for all $T$; Fig. 1(b) $(T \leq 0.3 \mathrm{~K})$ shows that this is indeed the case. It is the small curvature and its change of sign from negative to positive (Fermi gas behavior) together with the change of the slope of $C(T, P) / T$ vs $P$ from positive at $T<T_{+}$to negative at $T>T_{+}$that is the origin of the pointlike crossing region at $T_{+}$. For ${ }^{3} \mathrm{He}$ and heavy-electron liquids we can estimate $\Delta_{P_{0}}^{(n)}(P)$ at $T_{+}(P)$ from the linear specific heat coefficient $\gamma(P)$ (a zerotemperature quantity). Using $\partial^{2} \chi^{(n)} / \partial T^{2} \simeq \gamma^{(n)}(P)$, with $\gamma^{(n)}(X)=\partial^{n} \gamma / \partial X^{n}$, we obtain $\Delta_{P_{0}}^{(n)}(P) \simeq[(1-$ $\left.\left.P / P_{0}\right)^{n+1} /(n+1) !\right]\left[P_{0}^{n+1} \gamma^{(n+1)}\left(P_{0}\right) / \gamma\left(P_{0}\right)\right]$. For ${ }^{3} \mathrm{He}$ [2] (with $P_{0}=15$ bar, $P_{0}^{2} \gamma^{(2)}\left(P_{0}\right) / \gamma\left(P_{0}\right) \simeq 5 \times 10^{-2}$ ) we find $|W(P)| \lesssim 0.03$ for $0 \leq P \leq 30$ bar; i.e., on the scale of Fig. 1(a) the crossing region at $T_{+} \simeq 160 \mathrm{mK}$ is indeed essentially confined to a point. Similarly, for the crossing at $T_{+} \simeq 5 \mathrm{~K}$ in $\mathrm{CeAl}_{3}$ [6] [with $P_{0}=4.8 \mathrm{kbar}$, $\left.P_{0}^{2} \gamma^{(2)}\left(P_{0}\right) / \gamma\left(P_{0}\right) \simeq 0.4\right]$ we obtain $|W(P)| \lesssim 0.2$ for $P$ between $0.4-8.2 \mathrm{kbar}$, implying a rather narrow crossing region [Fig. 3(a)].

(ii) Linear $X$ dependence of $\chi^{(1)}$ : The situation is particularly clearcut if $\xi$ is a linear function of $X$, i.e., $\xi(T, X)=\chi^{(1)}(T) X$, as in linear-response theory. Then the crossing condition, Eq. (2), takes the form $d^{2} \chi\left(T_{+}\right) / d T^{2}=0$, where $T_{+}=T_{+}\left(X_{0}=0\right)$. This implies that $\Delta_{0}^{(n)}$ vanishes identically for $n \geq 1$. In this case all specific heat curves intersect exactly at one point. The width $\left|W_{0}(X)\right|$ becomes finite only through nonlinear terms in $\xi(T, X)=\chi^{(1)}(T) X+\frac{1}{3 !} \chi^{(3)}(T) X^{3}+\ldots$, where $\chi^{(n)}(T)=\chi^{(n)}(T, X=0)$. The lowest-order contribution to the width is given by $\Delta_{0}^{(3)}(X)$. For small enough $X$ (this depends on the system), i.e., in the linear regime, the specific heat curves must therefore necessarily cross at a well-defined point. This is seen to be the case in the specific heat curves $C(T, B)$ of the $d=1$ Hubbard model at $U=$ const [13] where $B$ is rather small, as well as in $\mathrm{CeCu}_{6-x} \mathrm{Au}_{x}[12,21]$ where the crossing region is only sharp for $B \lesssim 3 \mathrm{~T}$ [Fig. 3(b)]. The same arguments apply to $C(T, U)$ of the paramagnetic phase of Hubbard models $[4,14]$ where we now choose $\xi=\tilde{D}(T, U)=\frac{1}{4}-D(T, U)$ at half filling such that $\tilde{D}(T, 0)=0$. To a good approximation $\tilde{D}(T, U)$ is linear in $U$ for not too large $U$ at all temperatures [22]. We find $|W(U)| \lesssim 0.05$ for $U \lesssim 2.5 t^{*}$ in the $d=\infty$ Hubbard model [23].

In Hubbard models the intersection of $C(T, U) / k_{B}=$ $f(T / t, U / t)$ curves is sharp only at high temperatures. At low temperatures the generation of low-energy excitations leads to a renormalized energy scale $t \rightarrow t_{\text {eff }} \ll t$. Hence a perturbation expansion of $E(T, U)$ or $C(T, U)$ to second order in $U$ will be valid only for a small range of $U$ values, implying a wide crossing region, $|W(U)| \sim 1$, at low temperatures.

In summary, we showed that the remarkable crossing of specific heat curves $C(T, X)$ vs $T$ for different thermodynamic variables $X$, first observed in ${ }^{3} \mathrm{He}$ [2] and the Hubbard model [4], is not accidental but can be found in many correlated systems. The width of the crossing region is found to give explicit information about the temperature dependence of the generalized susceptibilities associated with $X$. A related observation, that for Hubbard models the value of the specific heat at the crossing point is almost universal, will be discussed elsewhere [24]. 
I am grateful to M. Kollar, G. Baskaran, D. S. Greywall, H. Keiter, H. v. Löhneysen, A. Loidl, W. Metzner, E. Müller-Hartmann, H. R. Ott, H. G. Schlager, F. Steglich, and G.R. Stewart for useful discussions, and to N. Blümer, N. Chandra, M. Kollar, R. Römer, and J. Schlipf for helping with the figures. I also thank R. A. Fisher, F. Gebhard, G. Georges, A. Klümper, H.v. Löhneysen, and G. R. Stewart for kindly providing me with data and figures of the specific heat of various correlated systems. This research was supported in part by the SFB 341 of the Deutsche Forschungsgemeinschaft, and the National Science Foundation under Grant No. PHY94-07194.

*New permanent address: Theoretische Physik III, Elektronische Korrelationen und Magnetismus, Universität Augsburg, 86135 Augsburg, Germany. Electronic address: vollha@physik.uni-augsburg.de

[1] D. F. Brewer, J. G. Daunt, and A. K. Sreedhar, Phys. Rev. 115, 836 (1959).

[2] D. S. Greywall, Phys. Rev. B 27, 2747 (1983). We plot his data as $C(T, P)$ with $P=P(V, T=0.1 \mathrm{~K})$. For $T \leq$ $0.5 \mathrm{~K}$ and $P \lesssim 30$ bar $C(T, V)$ and $C(T, P)$ of liquid ${ }^{3} \mathrm{He}$ differ by less than $0.2 \%$. We note that at low temperatures $C(T, P) / T$ vs $P$, but not $C(T, V) / T$ vs $V$, is almost a straight line for all $2.7 \mathrm{mK}<T<2.5 \mathrm{~K}$ (see below).

[3] K. Seiler, C. Gros, T. M. Rice, K. Ueda, and D. Vollhardt, J. Low Temp. Phys. 64, 195 (1986).

[4] A. Georges and W. Krauth, Phys. Rev. B 48, 7167 (1993).

[5] Figure 2 was calculated by R. Römer and J. Schlipf (unpublished) using the program of Georges and Krauth [4].

[6] G. E. Brodale, R. A. Fisher, N. E. Phillips, and J. Flouquet, Phys. Rev. Lett. 56, 390 (1986); R. A. Fisher (private communication). At $P=0$ a magnetic anomaly occurs; hence we neglect the $P=0$ curve.

[7] N.E. Phillips, R. A. Fisher, J. Flouquet, A. L. Giorgi, J. A. Olsen, and G. R. Stewart, J. Magn. Magn. Mater. 63 \& 64, 332 (1987).

[8] A. de Visser, J.C.P. Klaasse, M. van Sprang, J.J.M. Franse, A. Menovsky, and T.T.M. Palstra, J. Magn. Magn. Mater. 54-57, 375 (1986).

[9] F. Steglich, C. Geibel, K. Gloos, G. Olesch, C. Schank, C. Wassilew, A. Loidl, A. Krimmel, and G. R. Stewart, J. Low Temp. Phys. 95, 3 (1994). Except for $x=0$, where perhaps an anomaly occurs, the measured specific heat curves intersect at $\sim 2.9 \mathrm{~K}$.

[10] A. Germann and H. v. Löhneysen, Europhys. Lett. 9, 367 (1989).

[11] H.G. Schlager, A. Schröder, M. Welsch, and H.v. Löhneysen, J. Low Temp. Phys. 90, 181 (1993); (private communication).

[12] J. Fischer, A. Schröder, H. v. Löhneysen, W. Bauhofer, and U. Steigenberger, Phys. Rev. B 39, 11775 (1989).
[13] T. Usuki, N. Kawakami, and A. Okiji, J. Phys. Soc. Jpn. 59, 1357 (1990).

[14] F. Gebhard, A. Girndt, and A.E. Ruckenstein, Phys. Rev. B 49, 10926 (1994).

[15] In the Fermi gas $\partial C / \partial P<0$ for all $T$.

[16] With Eq. (1) the generalized expansion coefficient $\alpha(T, X)=\partial \ln \xi(T, X) / \partial T$ becomes $\alpha=-(d \gamma / d X) T /$ $\xi(0, X)>0$ at low enough temperatures, i.e., is negative when $d \gamma / d X>0$. K. A. Brueckner and K. R. Atkins [Phys. Rev. Lett. 1, 315 (1958)] attributed this anomalous behavior of $\alpha$ to the approach of momentum order in the limit $T \rightarrow 0$. They argued that momentum order would cause the liquid to expand to "make room" for the strongly interacting atoms. This would then explain $\partial C / \partial X>0$ both in ${ }^{3} \mathrm{He}$, heavy fermion systems and all Hubbard models. Unfortunately, the argument cannot be substantiated; in particular, it neither holds for spinless fermions, nor for the momentum-ordered phase of ${ }^{4} \mathrm{He}$.

[17] According to Greywall's data [2] Fermi liquid theory is valid in ${ }^{3} \mathrm{He}$ only below a temperature $T_{\mathrm{FL}}(P)$, with $T_{\mathrm{FL}}(0) \simeq 0.16 \mathrm{~K}$ to $T_{\mathrm{FL}}(30$ bar $) \simeq 0.1 \mathrm{~K}$.

[18] The Landau Fermi liquid regime sets in only at much lower temperatures [K. Andres, J.E. Graebner, and H. R. Ott, Phys. Rev. Lett. 35, 1979 (1975)].

[19] I am grateful to H. v. Löhneysen for drawing my attention to the importance of crystal field excitations at higher temperatures. See, for example, B. Stroka, A. Schröder, T. Trappmann, H. v. Löhneysen, M. Loewenhaupt, and A. Severing, Z. Phys. B 90, 155 (1993).

[20] J. Wilks, The Properties of Liquid and Solid Helium (Clarendon, Oxford, 1967).

[21] With $T_{+} \simeq 1.1 \mathrm{~K}$ and $\partial^{2} \chi^{(3)}\left(T_{+}\right) / \partial T^{2} \simeq-2 \times 10^{-3} \times$ [ $\left.\mu_{\mathrm{B}} / \mathrm{T}^{3} \mathrm{~K}^{2}\right]$, obtained from Fig. 5(a) of Ref. [11], we find $|W(B)| \lesssim 0.1$ for $B \lesssim 3 \mathrm{~T}$ along the $c$ direction.

[22] Up to $U \lesssim 2.5 t$, with $t$ as the hopping amplitude, the ground state energy $E_{0}(T, U)$ of the $d=1$ Hubbard model is very well described by second-order perturbation theory. Therefore $\tilde{D}=-\partial E_{0} / \partial U$ is almost linear up to this $U$ value. The same is true in $d=\infty$ [see Fig. 34 of the review by A. Georges, G. Kotliar, W. Krauth, and M. J. Rozenberg, Rev. Mod. Phys. 68, 13 (1996)]. At high temperatures $\tilde{D}(T, U)=\frac{1}{4} \tanh (U / 4 T)$ is almost linear, too, independent of dimension. This makes plausible why $\tilde{D}$ is linear in $U$ even at intermediate temperatures. For $d=\infty$ this is explicitly confirmed by the results in Fig. 6 of Ref. [4]. - We estimated $\partial^{5} \tilde{D} / \partial^{2} T \partial^{3} U$ in $d=\infty$, and hence $\Delta^{(1)}$, by interpolating the low- $T$ limit $\tilde{D}=\tilde{D}(U, 0)+\frac{1}{2} \gamma^{\prime}(U) T^{2}$ [with $\tilde{D}(U, 0)$ and $\gamma(U)$ taken from the Gutzwiller approximation] and the high- $T$ limit with a polynomial in $T$.

[23] The crossing of curves reported in Fig. 4 of Ref. [4] has a particularly small width, $|W(U)| \lesssim 0.004$, caused by an almost perfect $U^{2}$ dependence of $E(T, U)-U / 2$ up to $U=2.5 t^{*}$. It is not clear at present whether this is partly caused by the "iterated perturbation theory" itself, which iterates the second-order weak-coupling approximation to the self-energy.

[24] N. Chandra, M. Kollar, and D. Vollhardt (to be published). 